Supplemental Table 1. Hazard ratios (HRs) for incidence of coronary artery disease and cerebrovascular disease according to combinations of glucose status and systolic blood pressure categories and diastolic blood pressure categories among individuals with diabetes who excluding patients that took metformin and/or SGLT-2i at baseline

\begin{tabular}{|c|c|c|c|}
\hline Outcomes & $\begin{array}{c}\text { Diabetes } \\
\text { events / N }\end{array}$ & $\mathrm{HR}(95 \% \mathrm{CI})$ & P-Value \\
\hline \multicolumn{4}{|c|}{ Coronary artery disease } \\
\hline \multicolumn{4}{|c|}{ Systolic blood pressure } \\
\hline$\leq 119 \mathrm{mmHg}$ & $84 / 7,859$ & 1.00 (Ref.) & - \\
\hline $120-129 \mathrm{mmHg}$ & $142 / 7,795$ & $1.63(1.24-2.13)$ & $<0.001$ \\
\hline $130-139 \mathrm{mmHg}$ & $114 / 6,822$ & 1.48 (1.11-1.97) & 0.007 \\
\hline $140-149 \mathrm{mmHg}$ & $86 / 3,947$ & $1.88(1.39-2.56)$ & $<0.001$ \\
\hline$\geq 150 \mathrm{mmHg}$ & $109 / 3,500$ & $2.81(2.10-3.76)$ & $<0.001$ \\
\hline \multicolumn{4}{|c|}{ Diastolic blood pressure } \\
\hline$\leq 74 \mathrm{mmHg}$ & $102 / 8,653$ & 1.00 (Ref.) & - \\
\hline 75-79 mmHg & $84 / 4,800$ & $1.36(1.02-1.81)$ & 0.039 \\
\hline $80-84 \mathrm{mmHg}$ & $117 / 6,105$ & $1.44(1.10-1.88)$ & 0.008 \\
\hline $85-89 \mathrm{mmHg}$ & $83 / 4,298$ & $1.42(1.06-1.91)$ & 0.019 \\
\hline$\geq 90 \mathrm{mmHg}$ & $149 / 6,067$ & $1.91(1.47-2.48)$ & $<0.001$ \\
\hline
\end{tabular}

\title{
Cerebrovascular disease
}

Systolic blood pressure
$\leq 119 \mathrm{mmHg}$
$68 / 8,073 \quad 1.00$ (Ref.)
$120-129 \mathrm{mmHg}$
$109 / 8,017 \quad \mathbf{1 . 5 5}(\mathbf{1 . 1 5}-\mathbf{2 . 1 1}) \quad 0.005$
130-139 $\mathrm{mmHg}$
$91 / 6,973 \quad 1.43(1.04-1.96) \quad 0.029$
140-149 $\mathrm{mmHg}$
$67 / 3,981 \quad 1.78(\mathbf{1 . 2 6 - 2 . 5 1 )} \quad 0.001$
$\geq 150 \mathrm{mmHg}$
$107 / 3,572 \quad 3.32(\mathbf{2 . 4 3 - 4 . 5 3 )}<0.001$

Diastolic blood pressure
$\leq 74 \mathrm{mmHg}$
92 / 8,926 1.00 (Ref.)
$75-79 \mathrm{mmHg}$
$68 / 4,914 \quad 1.30(0.95-1.78) \quad 0.106$
80-84 mmHg
$76 / 6,264 \quad 1.11(0.82-1.51) \quad 0.490$
$85-89 \mathrm{mmHg}$
$66 / 4,372 \quad 1.38(1.00-1.90) \quad 0.051$ 


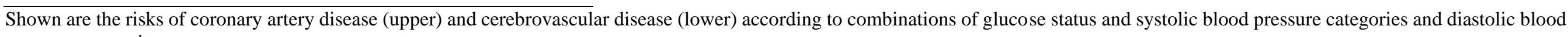
pressure categories.

Adjusted for age, sex, smoking, body mass index, low density lipoprotein cholesterol, high density lipoprotein cholesterol, and antihypertensive drug therapy.

Abbreviations: Border, borderline glycemia; HR, Hazard Ratio; NGT, normoglycemia. 
Supplemental Table 2. Hazard ratios (HRs) for the incidence of coronary artery disease and cerebrovascular disease according to combinations of glucose status and systolic blood pressure categories and diastolic blood pressure categories among older adults (age $\geq 65$ )

\begin{tabular}{|c|c|c|c|c|c|c|c|c|c|}
\hline \multirow[t]{2}{*}{ Outcomes } & \multirow{2}{*}{$\begin{array}{c}\text { NGT } \\
\text { events / N }\end{array}$} & \multirow[b]{2}{*}{$\mathrm{HR}(95 \% \mathrm{CI})$} & \multirow[b]{2}{*}{ P-Value } & \multirow{2}{*}{$\begin{array}{c}\text { Border } \\
\text { events / N }\end{array}$} & \multirow[b]{2}{*}{$\mathrm{HR}(95 \% \mathrm{CI})$} & \multirow[b]{2}{*}{ P-Value } & \multirow{2}{*}{$\begin{array}{c}\text { Diabetes } \\
\text { events / N }\end{array}$} & \multirow[b]{2}{*}{$\mathrm{HR}(95 \% \mathrm{CI})$} & \multirow[b]{2}{*}{ P-Value } \\
\hline & & & & & & & & & \\
\hline \multicolumn{10}{|c|}{ Coronary artery disease } \\
\hline \multicolumn{10}{|c|}{ Systolic blood pressure } \\
\hline$\leq 119 \mathrm{mmHg}$ & $11 / 1,198$ & 1.00 (Ref.) & - & $14 / 1,568$ & 1.00 (Ref.) & - & $11 / 478$ & 1.00 (Ref.) & - \\
\hline $120-129 \mathrm{mmHg}$ & $9 / 947$ & $0.90(0.37-2.21)$ & 0.818 & $17 / 1,291$ & $1.35(0.66-2.76)$ & 0.406 & $16 / 544$ & $1.27(0.58-2.74)$ & 0.549 \\
\hline $130-139 \mathrm{mmHg}$ & $8 / 754$ & $0.95(0.37-2.44)$ & 0.916 & $26 / 1,218$ & $2.12(1.09-4.10)$ & 0.026 & $8 / 485$ & $0.79(0.31-2.00)$ & 0.620 \\
\hline $140-149 \mathrm{mmHg}$ & $5 / 370$ & $1.15(0.38-3.49)$ & 0.811 & $11 / 692$ & $1.44(0.65-3.22)$ & 0.371 & $5 / 314$ & $0.72(0.25-2.10)$ & 0.549 \\
\hline$\geq 150 \mathrm{mmHg}$ & $1 / 332$ & NA & - & $2 / 594$ & NA & - & $7 / 315$ & $0.99(0.37-2.62)$ & 0.983 \\
\hline \multicolumn{10}{|c|}{ Diastolic blood pressure } \\
\hline$\leq 74 \mathrm{mmHg}$ & $13 / 1,645$ & 1.00 (Ref.) & - & $21 / 2,242$ & 1.00 (Ref.) & - & $26 / 950$ & 1.00 (Ref.) & - \\
\hline $75-79 \mathrm{mmHg}$ & $9 / 640$ & $1.47(0.63-3.47)$ & 0.373 & 18 / 946 & $1.78(0.94-3.36)$ & 0.076 & $9 / 355$ & $0.95(0.44-2.04)$ & 0.895 \\
\hline $80-84 \mathrm{mmHg}$ & $5 / 583$ & $0.87(0.31-2.45)$ & 0.787 & 14 / 984 & $1.30(0.65-2.57)$ & 0.455 & $4 / 352$ & NA & - \\
\hline $85-89 \mathrm{mmHg}$ & $5 / 379$ & $1.20(0.42-3.46)$ & 0.732 & $10 / 538$ & $1.60(0.74-3.44)$ & 0.231 & $4 / 233$ & NA & - \\
\hline$\geq 90 \mathrm{mmHg}$ & $2 / 354$ & NA & - & $7 / 653$ & $0.90(0.38-2.16)$ & 0.821 & $4 / 246$ & NA & - \\
\hline \multicolumn{10}{|c|}{ Cerebrovascular disease } \\
\hline \multicolumn{10}{|c|}{ Systolic blood pressure } \\
\hline$\leq 119 \mathrm{mmHg}$ & $13 / 1,229$ & 1.00 (Ref.) & - & $26 / 1,613$ & 1.00 (Ref.) & - & $15 / 516$ & 1.00 (Ref.) & - \\
\hline $120-129 \mathrm{mmHg}$ & $13 / 962$ & $1.23(0.56-2.67)$ & 0.603 & $23 / 1,326$ & $0.98(0.56-1.72)$ & 0.943 & $17 / 576$ & $1.02(0.51-2.06)$ & 0.949 \\
\hline $130-139 \mathrm{mmHg}$ & $15 / 774$ & $1.75(0.82-3.73)$ & 0.151 & $25 / 1,232$ & $1.11(0.64-1.94)$ & 0.713 & $16 / 503$ & $1.23(0.60-2.52)$ & 0.579 \\
\hline $140-149 \mathrm{mmHg}$ & $2 / 378$ & NA & - & 19 / 697 & $1.40(0.77-2.56)$ & 0.269 & $13 / 330$ & $1.43(0.67-3.04)$ & 0.359 \\
\hline$\geq 150 \mathrm{mmHg}$ & $6 / 338$ & $1.65(0.62-4.41)$ & 0.319 & $16 / 597$ & $1.46(0.77-2.76)$ & 0.241 & $9 / 330$ & $1.01(0.44-2.35)$ & 0.977 \\
\hline \multicolumn{10}{|c|}{ Diastolic blood pressure } \\
\hline$\leq 74 \mathrm{mmHg}$ & $20 / 1,674$ & 1.00 (Ref.) & - & $44 / 2,305$ & 1.00 (Ref.) & - & $32 / 1,016$ & 1.00 (Ref.) & - \\
\hline 75-79 mmHg & $7 / 663$ & $0.83(0.35-1.98)$ & 0.678 & $13 / 969$ & $0.64(0.35-1.20)$ & 0.167 & $11 / 372$ & $1.01(0.51-2.01)$ & 0.979 \\
\hline
\end{tabular}




\begin{tabular}{lccccccccc}
\hline $80-84 \mathrm{mmHg}$ & $10 / 600$ & $1.30(0.60-2.80)$ & 0.502 & $22 / 985$ & $1.06(0.63-1.77)$ & 0.838 & $11 / 371$ & $0.97(0.48-1.93)$ & 0.924 \\
$85-89 \mathrm{mmHg}$ & $5 / 386$ & $0.96(0.36-2.60)$ & 0.940 & $6 / 536$ & $0.50(0.21-1.19)$ & 0.118 & $9 / 249$ & $1.25(0.59-2.65)$ & 0.561 \\
$\geq 90 \mathrm{mmHg}$ & $7 / 358$ & $1.50(0.62-3.60)$ & 0.368 & $24 / 670$ & $1.68(1.01-2.81)$ & 0.045 & $7 / 247$ & $0.99(0.43-2.27)$ & 0.983 \\
\hline
\end{tabular}

Shown are the risks of coronary artery disease (upper) and cerebrovascular disease (lower) according to combinations of glucose status and systolic blood pressure categories and diastolic blood pressure categories.

Adjusted for age, sex, smoking, body mass index, low density lipoprotein cholesterol, high density lipoprotein cholesterol, and antihypertensive drug therapy.

Abbreviations: Border, borderline glycemia; HR, Hazard Ratio; NGT, normoglycemia. 
Supplemental Table 3. Hazard ratios (HRs) for the incidence of coronary artery disease and cerebrovascular disease according to combinations of glucose status and systolic blood pressure categories and diastolic blood pressure categories

\begin{tabular}{|c|c|c|c|c|c|c|c|c|c|}
\hline \multirow[t]{2}{*}{ Outcomes } & \multicolumn{3}{|l|}{ NGT } & \multicolumn{3}{|l|}{ Border } & \multicolumn{3}{|l|}{ Diabetes } \\
\hline & events / N & HR (95\% CI) & P-Value & events / N & HR $(95 \%$ CI) & P-Value & events / N & HR $(95 \%$ CI) & P-Value \\
\hline \multicolumn{10}{|c|}{ Coronary artery disease } \\
\hline \multicolumn{10}{|c|}{ Systolic blood pressure } \\
\hline$\leq 119 \mathrm{mmHg}$ & $182 / 233,180$ & 1.00 (Ref.) & - & $191 / 77,526$ & $1.49(1.22-1.83)$ & $<0.001$ & $115 / 9,560$ & $3.72(2.93-4.73)$ & $<0.001$ \\
\hline $120-129 \mathrm{mmHg}$ & $236 / 84,828$ & $2.18(1.79-2.64)$ & $<0.001$ & $210 / 44,742$ & $2.11(1.73-2.59)$ & $<0.001$ & $184 / 9,680$ & $5.42(4.37-6.72)$ & $<0.001$ \\
\hline $130-139 \mathrm{mmHg}$ & 169 / 40,959 & $2.55(2.07-3.15)$ & $<0.001$ & $200 / 28,618$ & $2.74(2.22-3.36)$ & $<0.001$ & $158 / 8,365$ & $5.07(4.05-6.35)$ & $<0.001$ \\
\hline $140-149 \mathrm{mmHg}$ & $87 / 13,542$ & $3.39(2.62-4.40)$ & $<0.001$ & $101 / 12,444$ & $2.92(2.28-3.76)$ & $<0.001$ & $118 / 4,789$ & $6.26(4.90-7.99)$ & $<0.001$ \\
\hline$\geq 150 \mathrm{mmHg}$ & $59 / 8,303$ & $3.68(2.73-4.95)$ & $<0.001$ & $94 / 8,821$ & $3.89(3.01-5.02)$ & $<0.001$ & $136 / 4,144$ & $8.39(6.62-10.63)$ & $<0.001$ \\
\hline \multicolumn{10}{|c|}{ Diastolic blood pressure } \\
\hline$\leq 74 \mathrm{mmHg}$ & $184 / 236,838$ & 1.00 (Ref.) & - & $187 / 77,320$ & $1.47(1.20-1.81)$ & $<0.001$ & $139 / 10,674$ & $3.86(3.07-4.85)$ & $<0.001$ \\
\hline $75-79 \mathrm{mmHg}$ & $115 / 51,366$ & $1.66(1.32-2.10)$ & $<0.001$ & $124 / 27,087$ & $1.95(1.55-2.46)$ & $<0.001$ & 114 / 5,966 & $5.03(3.95-6.41)$ & $<0.001$ \\
\hline $80-84 \mathrm{mmHg}$ & $153 / 45,464$ & $2.06(1.66-2.55)$ & $<0.001$ & $163 / 28,832$ & $2.15(1.74-2.67)$ & $<0.001$ & $154 / 7,512$ & $5.14(4.10-6.44)$ & $<0.001$ \\
\hline $85-89 \mathrm{mmHg}$ & $123 / 23,647$ & $2.82(2.24-3.55)$ & $<0.001$ & $113 / 17,777$ & $2.21(1.74-2.81)$ & $<0.001$ & $113 / 5,215$ & $5.23(4.09-6.69)$ & $<0.001$ \\
\hline$\geq 90 \mathrm{mmHg}$ & $158 / 23,497$ & $3.32(2.68-4.13)$ & $<0.001$ & $209 / 21,135$ & $3.37(2.74-4.15)$ & $<0.001$ & $191 / 7,171$ & $6.55(5.26-8.14)$ & $<0.001$ \\
\hline \multicolumn{10}{|c|}{ Cerebrovascular disease } \\
\hline \multicolumn{10}{|c|}{ Systolic blood pressure } \\
\hline$\leq 119 \mathrm{mmHg}$ & $511 / 234,122$ & 1.00 (Ref.) & - & $273 / 78,073$ & $1.09(0.94-1.27)$ & 0.242 & $81 / 9,864$ & $1.68(1.32-2.13)$ & $<0.001$ \\
\hline $120-129 \mathrm{mmHg}$ & $348 / 85,195$ & $1.48(1.29-1.70)$ & $<0.001$ & $315 / 45,111$ & $1.86(1.61-2.15)$ & $<0.001$ & $146 / 9,968$ & $2.84(2.33-3.45)$ & $<0.001$ \\
\hline $130-139 \mathrm{mmHg}$ & $306 / 41,121$ & $2.28(1.97-2.64)$ & $<0.001$ & $248 / 28,823$ & $2.05(1.75-2.40)$ & $<0.001$ & $119 / 8,564$ & $2.52(2.04-3.12)$ & $<0.001$ \\
\hline $140-149 \mathrm{mmHg}$ & $153 / 13,557$ & $3.10(2.58-3.73)$ & $<0.001$ & $146 / 12,512$ & $2.60(2.15-3.15)$ & $<0.001$ & $90 / 4,866$ & $3.20(2.53-4.05)$ & $<0.001$ \\
\hline$\geq 150 \mathrm{mmHg}$ & $160 / 8,337$ & $5.00(4.17-6.01)$ & $<0.001$ & $178 / 8,857$ & $4.44(3.71-5.32)$ & $<0.001$ & $133 / 4,226$ & $5.59(4.54-6.87)$ & $<0.001$ \\
\hline \multicolumn{10}{|c|}{ Diastolic blood pressure } \\
\hline$\leq 74 \mathrm{mmHg}$ & $503 / 23,7761$ & 1.00 (Ref.) & - & $282 / 77,938$ & $1.15(0.99-1.34)$ & 0.059 & $118 / 11,039$ & $2.12(1.72-2.62)$ & $<0.001$ \\
\hline 75-79 mmHg & $197 / 51,620$ & $1.41(1.20-1.67)$ & $<0.001$ & $144 / 27,250$ & $1.44(1.19-1.74)$ & $<0.001$ & $91 / 6,125$ & $2.85(2.26-3.60)$ & $<0.001$ \\
\hline $80-84 \mathrm{mmHg}$ & 245 / 45,632 & $1.78(1.52-2.08)$ & $<0.001$ & $236 / 29,002$ & $2.06(1.75-2.43)$ & $<0.001$ & $104 / 7,728$ & $2.54(2.03-3.17)$ & $<0.001$ \\
\hline
\end{tabular}




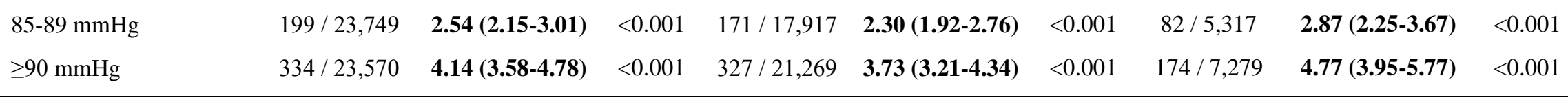

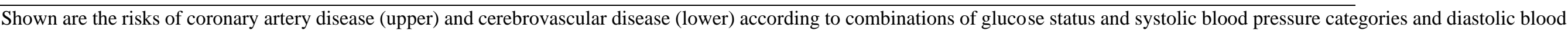
pressure categories.

Adjusted for age, sex, smoking, body mass index, low density lipoprotein cholesterol, high density lipoprotein cholesterol, and antihypertensive drug therapy.

Abbreviations: Border, borderline glycemia; HR, Hazard Ratio; NGT, normoglycemia. 
Supplemental Table 4. HRs for initiation of coronary artery disease and cerebrovascular disease according to combinations of glucose status and systolic blood pressure categories and diastolic blood pressure categories

\begin{tabular}{|c|c|c|c|c|c|c|c|c|c|}
\hline \multirow[t]{2}{*}{ Outcomes } & \multirow{2}{*}{$\begin{array}{c}\text { NGT } \\
\text { events / N }\end{array}$} & \multirow[b]{2}{*}{$\mathrm{HR}(95 \% \mathrm{CI})$} & \multirow[b]{2}{*}{ P-Value } & \multirow{2}{*}{$\begin{array}{c}\text { Border } \\
\text { events / N }\end{array}$} & \multirow[b]{2}{*}{ HR $(95 \%$ CI $)$} & \multirow[b]{2}{*}{ P-Value } & \multirow{2}{*}{$\begin{array}{c}\text { Diabetes } \\
\text { events / N }\end{array}$} & \multirow[b]{2}{*}{$\mathrm{HR}(95 \% \mathrm{CI})$} & \multirow[b]{2}{*}{ P-Value } \\
\hline & & & & & & & & & \\
\hline \multicolumn{10}{|c|}{ Coronary artery disease } \\
\hline \multicolumn{10}{|c|}{ Systolic blood pressure } \\
\hline$\leq 119 \mathrm{mmHg}$ & $182 / 233,180$ & 1.00 (Ref.) & - & $191 / 77,526$ & $1.32(1.07-1.63)$ & 0.010 & $115 / 9,560$ & $2.90(2.24-3.75)$ & $<0.001$ \\
\hline $120-129 \mathrm{mmHg}$ & $236 / 84,828$ & 1.00 (Ref.) & - & $210 / 44,742$ & $0.96(0.79-1.16)$ & 0.665 & 184 / 9,680 & $2.42(1.96-3.00)$ & $<0.001$ \\
\hline $130-139 \mathrm{mmHg}$ & $169 / 40,959$ & 1.00 (Ref.) & - & $200 / 28,618$ & $1.08(0.88-1.33)$ & 0.474 & $158 / 8,365$ & $2.04(1.61-2.58)$ & $<0.001$ \\
\hline $140-149 \mathrm{mmHg}$ & $87 / 13,542$ & 1.00 (Ref.) & - & $101 / 12,444$ & $0.94(0.70-1.26)$ & 0.683 & $118 / 4,789$ & $2.19(1.63-2.95)$ & $<0.001$ \\
\hline$\geq 150 \mathrm{mmHg}$ & $59 / 8,303$ & 1.00 (Ref.) & - & 94 / 8,821 & $1.16(0.83-1.61)$ & 0.375 & $136 / 4,144$ & 2.67 (1.93-3.69) & $<0.001$ \\
\hline \multicolumn{10}{|c|}{ Diastolic blood pressure } \\
\hline$\leq 74 \mathrm{mmHg}$ & 184 / 236,838 & 1.00 (Ref.) & - & $187 / 77,320$ & $1.26(1.02-1.56)$ & 0.032 & $139 / 10,674$ & $2.73(2.12-3.50)$ & $<0.001$ \\
\hline $75-79 \mathrm{mmHg}$ & $115 / 51,366$ & 1.00 (Ref.) & - & $124 / 27,087$ & $1.15(0.89-1.50)$ & 0.281 & $114 / 5,966$ & $2.97(2.23-3.96)$ & $<0.001$ \\
\hline $80-84 \mathrm{mmHg}$ & $153 / 45,464$ & 1.00 (Ref.) & - & $163 / 28,832$ & $1.07(0.86-1.34)$ & 0.547 & $154 / 7,512$ & $2.60(2.03-3.32)$ & $<0.001$ \\
\hline $85-89 \mathrm{mmHg}$ & $123 / 23,647$ & 1.00 (Ref.) & - & $113 / 17,777$ & $0.81(0.63-1.06)$ & 0.123 & $113 / 5,215$ & $1.97(1.49-2.61)$ & $<0.001$ \\
\hline$\geq 90 \mathrm{mmHg}$ & $158 / 23,497$ & 1.00 (Ref.) & - & $209 / 21,135$ & $1.11(0.90-1.37)$ & 0.342 & $191 / 7,171$ & $2.30(1.84-2.89)$ & $<0.001$ \\
\hline \multicolumn{10}{|c|}{ Cerebrovascular disease } \\
\hline \multicolumn{10}{|c|}{ Systolic blood pressure } \\
\hline$\leq 119 \mathrm{mmHg}$ & $511 / 234,122$ & 1.00 (Ref.) & - & $273 / 78,073$ & $1.02(0.88-1.19)$ & 0.786 & $81 / 9,864$ & $1.33(1.03-1.73)$ & 0.029 \\
\hline $120-129 \mathrm{mmHg}$ & 348 / 85,195 & 1.00 (Ref.) & - & $315 / 45,111$ & $1.20(1.03-1.41)$ & 0.021 & $146 / 9,968$ & $1.75(1.41-2.17)$ & $<0.001$ \\
\hline $130-139 \mathrm{mmHg}$ & $306 / 41,121$ & 1.00 (Ref.) & - & $248 / 28,823$ & $0.89(0.75-1.05)$ & 0.176 & $119 / 8,564$ & $1.10(0.87-1.39)$ & 0.412 \\
\hline $140-149 \mathrm{mmHg}$ & $153 / 13,557$ & 1.00 (Ref.) & - & $146 / 12,512$ & $0.92(0.73-1.16)$ & 0.480 & $90 / 4,866$ & $1.27(0.96-1.68))$ & 0.100 \\
\hline$\geq 150 \mathrm{mmHg}$ & $160 / 8,337$ & 1.00 (Ref.) & - & $178 / 8,857$ & $0.96(0.77-1.20)$ & 0.720 & $133 / 4,226$ & $1.33(1.04-1.71)$ & 0.025 \\
\hline \multicolumn{10}{|c|}{ Diastolic blood pressure } \\
\hline$\leq 74 \mathrm{mmHg}$ & $503 / 237,761$ & 1.00 (Ref.) & - & $282 / 77,938$ & $1.04(0.89-1.21)$ & 0.606 & $118 / 11,039$ & $1.61(1.28-2.03)$ & $<0.001$ \\
\hline 75-79 mmHg & $197 / 51,620$ & 1.00 (Ref.) & - & $144 / 27,250$ & $0.98(0.78-1.22)$ & 0.858 & $91 / 6,125$ & $1.76(1.33-2.33)$ & $<0.001$ \\
\hline $80-84 \mathrm{mmHg}$ & $245 / 45,632$ & 1.00 (Ref.) & - & $236 / 29,002$ & $1.18(0.98-1.42)$ & 0.077 & $104 / 7,728$ & 1.45 (1.13-1.87) & 0.004 \\
\hline
\end{tabular}




\begin{tabular}{|c|c|c|c|c|c|c|c|c|c|}
\hline $85-89 \mathrm{mmHg}$ & 199 / 23,749 & 1.00 (Ref.) & & $171 / 17,917$ & $0.97(0.78-1.19)$ & 0.745 & $82 / 5,317$ & $1.31(0.99-1.73)$ & 0.060 \\
\hline $90 \mathrm{mmHg}$ & $334 / 23,570$ & 1.00 (Ref.) & - & $327 / 21,269$ & $0.96(0.82-1.12)$ & 0.608 & $174 / 7,279$ & $1.30(1.07-1.59)$ & 0.010 \\
\hline
\end{tabular}

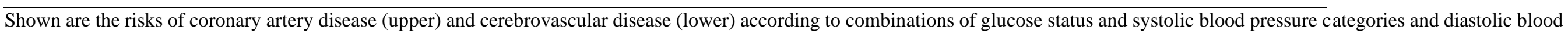
pressure categories.

Adjusted for age, sex, smoking, body mass index, low density lipoprotein cholesterol, high density lipoprotein cholesterol, and antihypertensive drug therapy.

Abbreviations: Border, borderline glycemia; HR, Hazard Ratio; NGT, normoglycemia 
Supplemental Figure 1. The incidence of coronary artery disease and cerebrovascular disease according to combinations of glucose status and systolic blood pressure categories and diastolic blood pressure categories

(A)

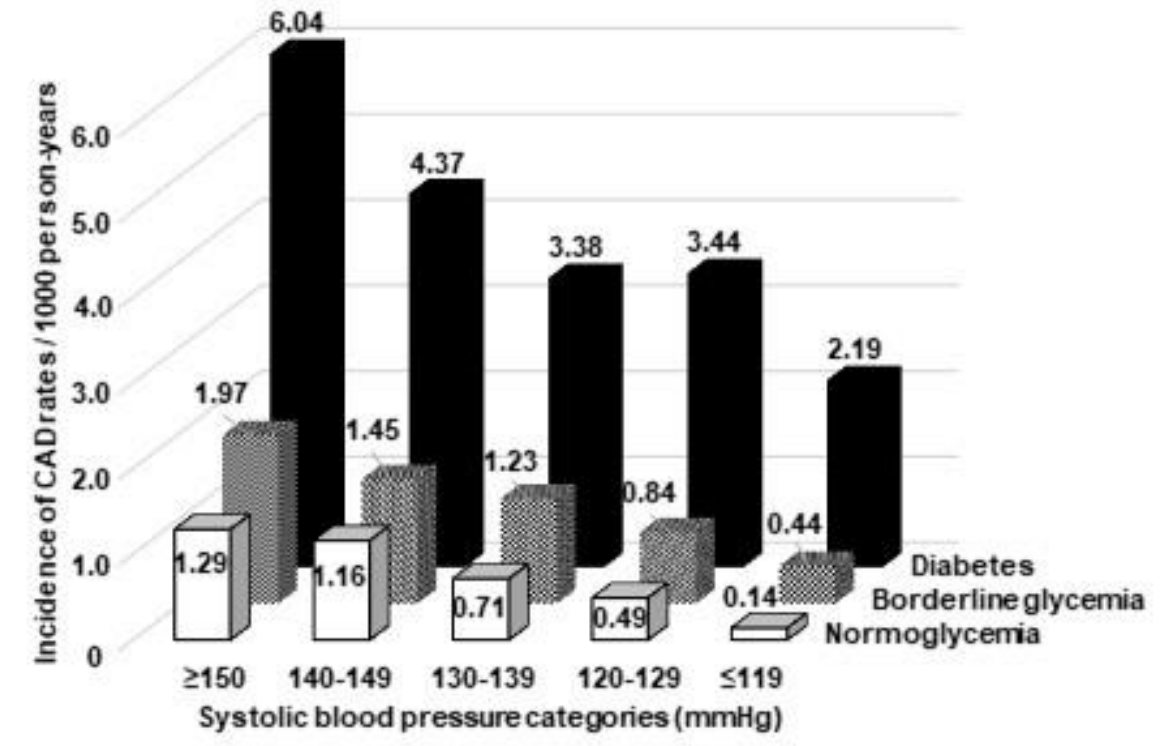

(C)

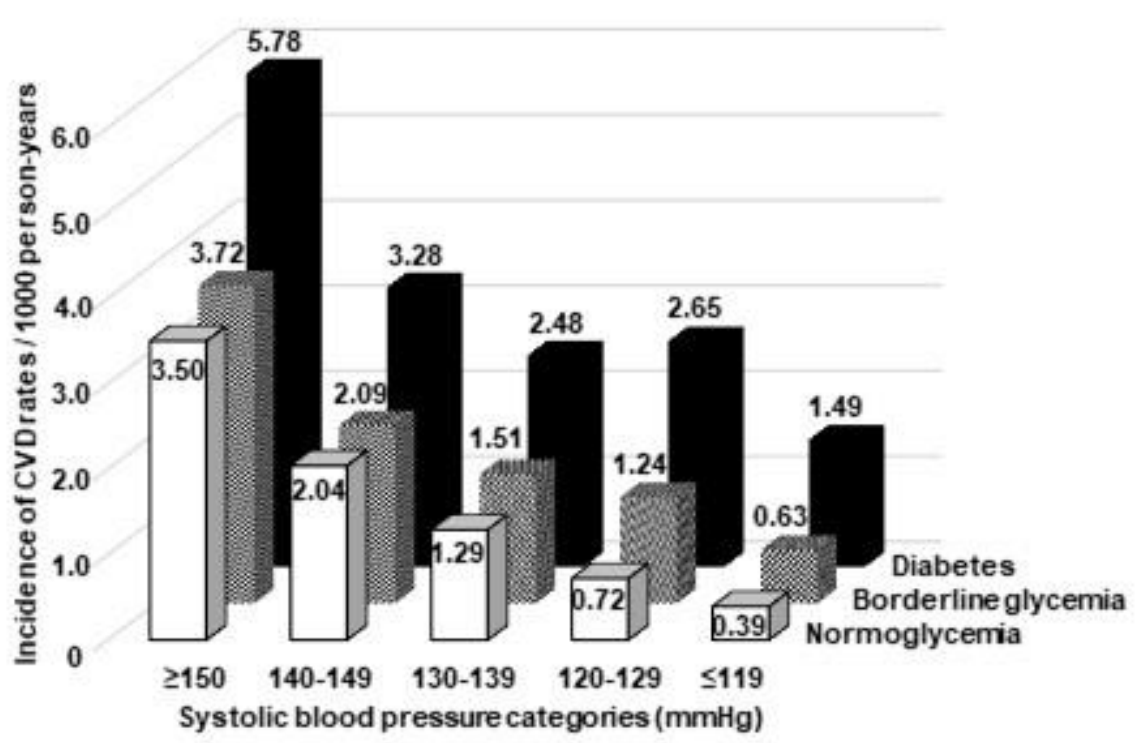

(D)
(B)
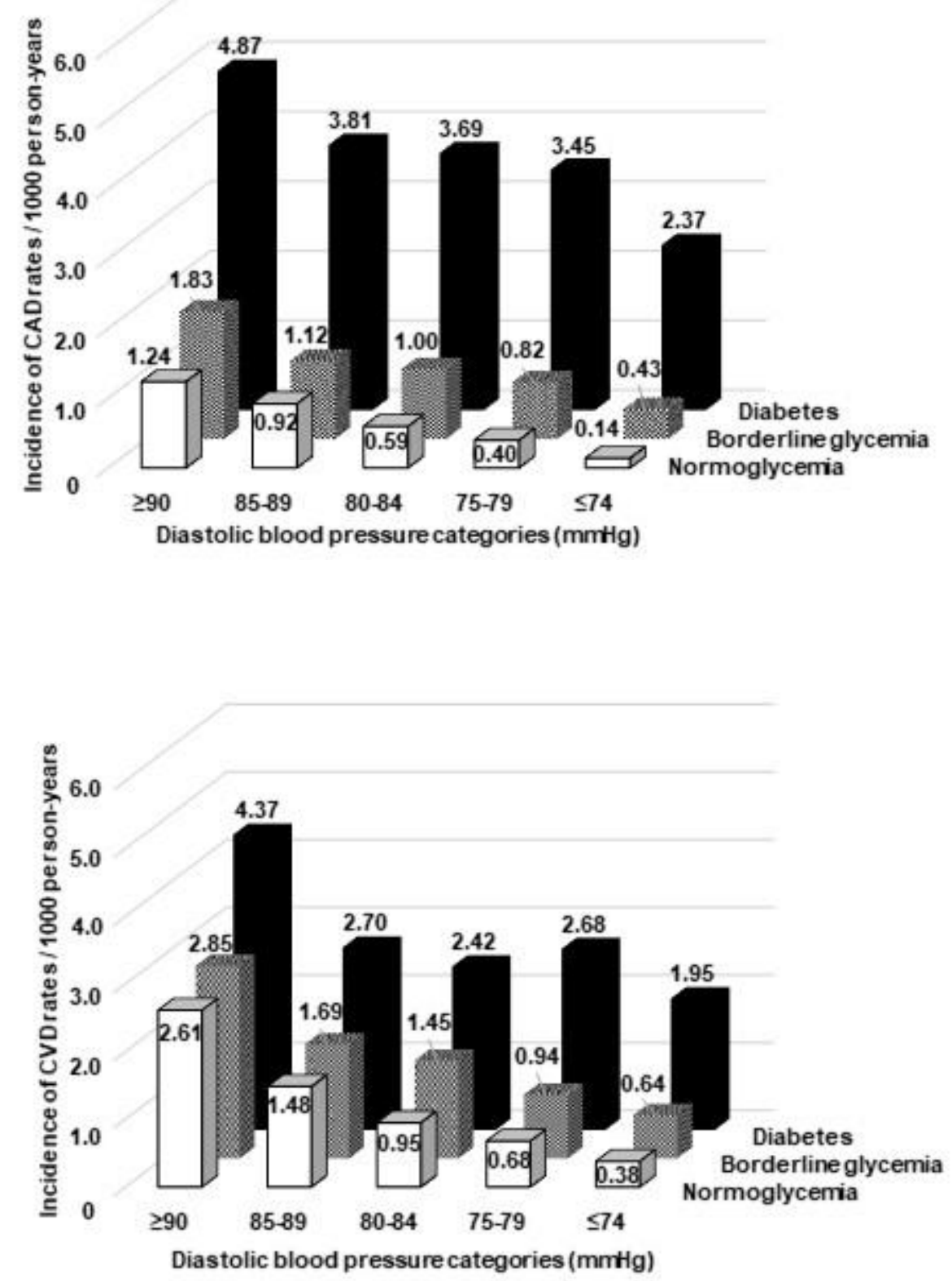


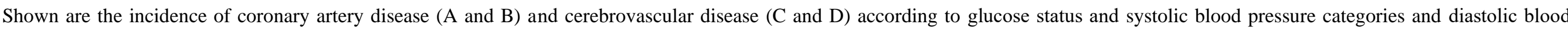
pressure categories.

Abbreviations: CAD, coronary artery disease; CVD, cerebrovascular disease. 


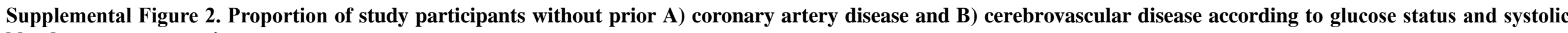
blood pressure categories

$\begin{array}{ll}\text { A) Without prior coronary artery disease } & \text { B) Without prior cerebrovascular disease }\end{array}$

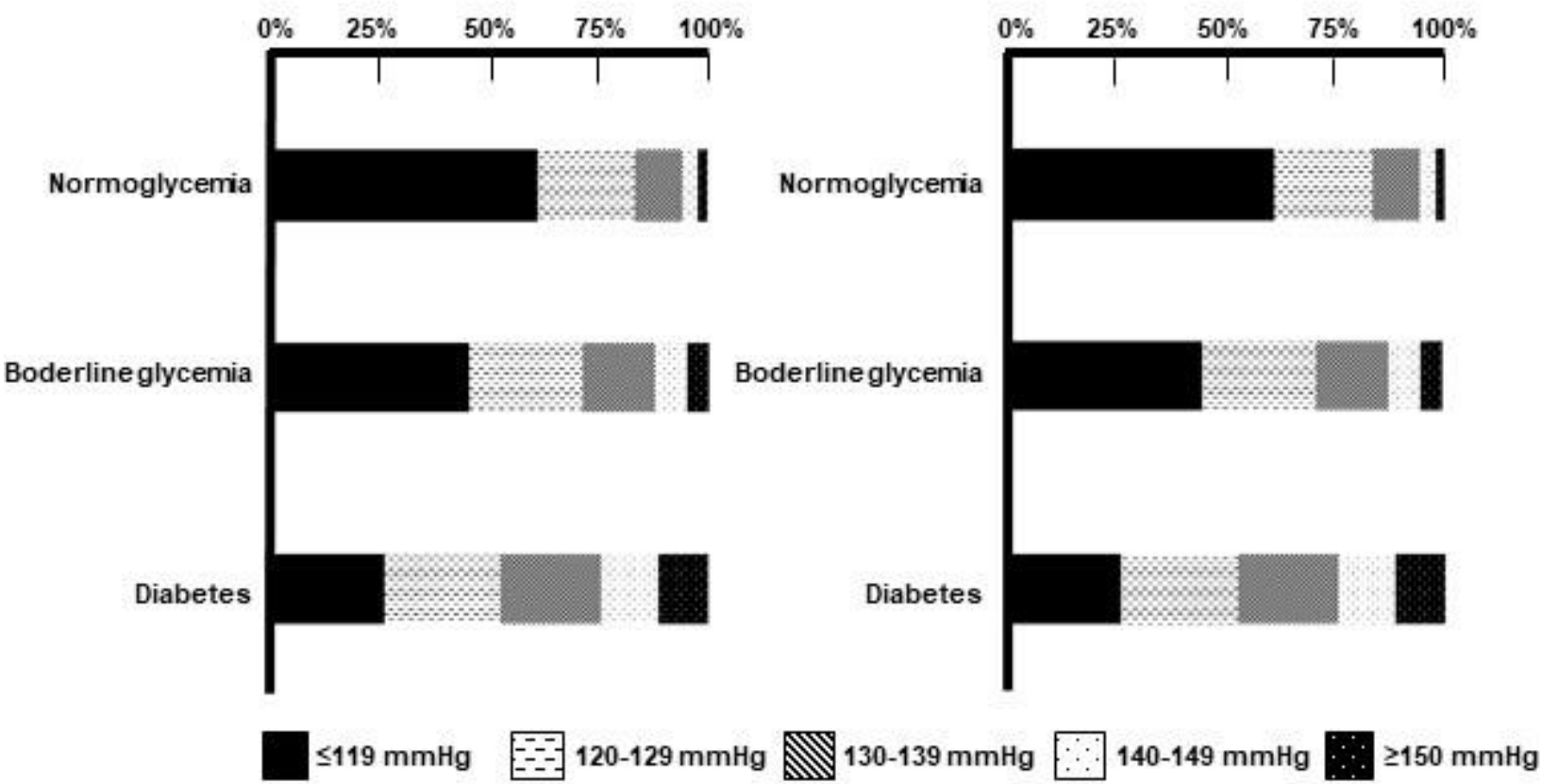

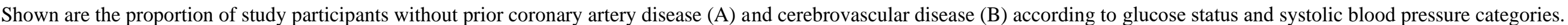

